\title{
Some Examples of Minimal Cantor Sets for Iterated Function Systems with Overlap
}

\author{
Katsutoshi SHINOHARA \\ Tokyo Metropolitan University \\ (Communicated by T. Soma)
}

\begin{abstract}
We give some examples of iterated function systems (IFSs) with overlap on the interval such that the semigroup action they give rise to has a minimal set homeomorphic to the Cantor set.
\end{abstract}

\section{Introduction}

In [2], the minimality of semigroup actions (equivalently, minimal set of iterated function systems, abbreviated as IFSs) on the interval are discussed and several examples of nonminimal actions are presented there. For these examples, we certainly know that the action is not minimal but we could not decide the "shape" of the minimal set (similar type of questions are extensively studied recently, see for example [4] for complex dynamical systems case).

In the case of group actions on the circle, there is a famous trichotomy of the minimal set (see for example Chapter 2 of [3]): Either it is a finite set, equal to the whole manifold, or homeomorphic to the Cantor set. Thus once we know that the minimal set is neither the finite orbit nor the whole manifold, then we can immediately conclude that it is homeomorphic to the Cantor set. On the other hand, in the case of semigroup actions, because of the lack of homogeneity of the minimal set, this trichotomy is no longer valid. The best thing we know in general is, as is stated in [1] (see Theorem 5.2 in [1]), the trichotomy of the following type: the minimal set is either a finite set, homeomorphic to the Cantor set or a closed set with non-empty interior. This trichotomy is not enough to conclude the type of minimal set just from the non-minimality of the action.

In this article, we give an example of class of IFSs with a minimal set homeomorphic to the Cantor set. They are produced by performing some modifications on the example presented in [2]. Thus, still the author does not know the type of minimal set for the original examples, see also Remark 2.

After the announcement of this example, Masayuki Asaoka (Kyoto University) showed me another example of minimal Cantor set using the measure theoretic argument. In the

Received April 22, 2013; revised August 30, 2013

Mathematics Subject Classification: 37B05, 37E05, 28A80

Key words and phrases: IFS with overlap, Cantor set, semigroup action, minimality 
appendix we present it.

\section{Axiomatic description of the example}

In this section, we prepare some definitions, give the axiomatic description of the example and the precise statement of our main result.

2.1. Overlapping. We consider IFSs on the closed unit interval $I:=[0,1]$ generated by two maps $f, g: I \rightarrow I$ satisfying the following conditions:

- $f, g: I \rightarrow I$ are $C^{1}$-diffeomorphisms on their images.

- $f(0)=0$ and $g(1)=1$.

- $f(x)<x<g(x)$ for $x \in(0,1)$.

- $0<g(0)<f(1)<1$.

We denote the set of the pairs of diffeomorphisms satisfying these conditions by $\mathcal{A} \subset$ $\left(\operatorname{Diff}_{\mathrm{im}}^{1}(I)\right)^{2}$ (by Diff ${ }_{\mathrm{im}}^{1}(I)$ we denote the set of $C^{1}$-maps from $I$ to itself which is a diffeomorphism on its image).

We prepare some notations and basic definitions. We denote the semigroup generated by $f$ and $g$ by $\langle f, g\rangle_{+}$. It acts on $I$ in a natural way. For $x \in I$, the orbit of $x$, denoted by $\mathcal{O}_{+}(x)$, is defined to be the set $\left\{\phi(x) \mid \phi \in\langle f, g\rangle_{+}\right\}$. A non-empty set $K \subset I$ is called a minimal set if for every $x \in K, \overline{\mathcal{O}_{+}(x)}=K$, where $\bar{X}$ denotes the closure of $X$. Finally, we say that the action of $\langle f, g\rangle_{+}$is minimal if $I$ is the minimal set, in other words, every point in $I$ has a dense orbit.

For IFSs generated by $(f, g) \in \mathcal{A}$, we can prove the following (see Lemma 1 in [2]):

Proposition 1. There exists a unique minimal set $K$. Furthermore, we have $K=$ $\overline{\mathcal{O}_{+}(0)}=\overline{\mathcal{O}_{+}(1)}$

For $(f, g) \in \mathcal{A}$, put $W:=[g(0), f(1)]$ and call it overlapping region ( $W$ is the overlap between the two intervals $f(I)$ and $g(I))$. Note that by the definition of $\mathcal{A}, W$ is an interval with non-empty interior.

2.2. Alignment of fundamental domains. We define two sequences of intervals $\left\{F_{n}\right\}$ and $\left\{G_{n}\right\}$ as follows:

- $F_{n}:=\left[f^{n+1}(1), f^{n}(1)\right]$.

- $G_{n}:=\left[g^{n}(0), g^{n+1}(0)\right]$.

Let us consider the following property for $(f, g)$ :

$$
W=f(I) \cap g(I) \text { is contained in the interior of } F_{1} \cup G_{1} .
$$

We call this property the single overlapping property and denote it by So. Note that this condition is equivalent to $f^{2}(1)<g(0)$ and $f(1)<g^{2}(0)$. This implies that So is a $C^{0}$-open property in $\mathcal{A}$. 

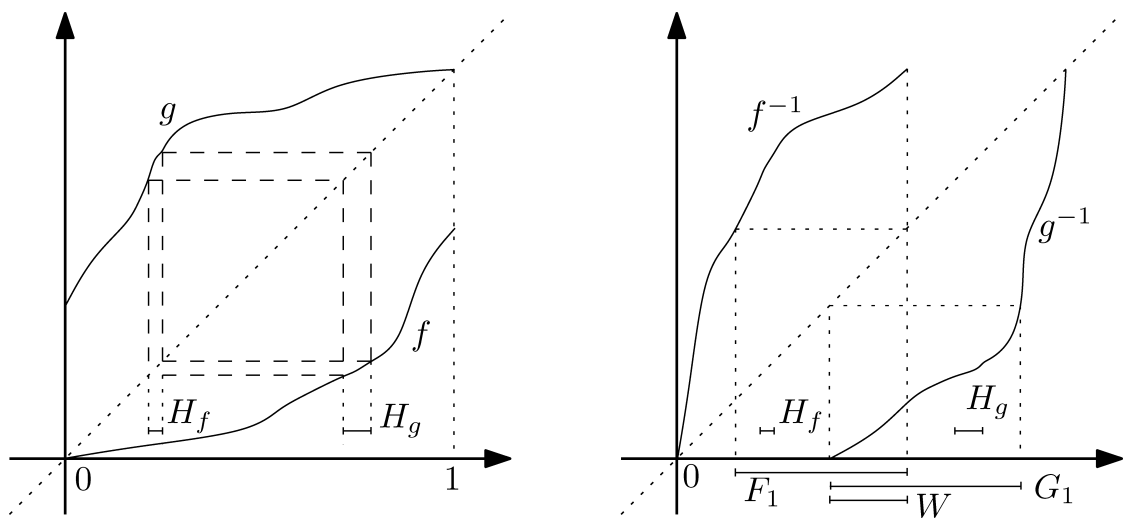

FIgURE 1. An example of IFS in $\mathcal{A}$ with So and Ho

2.3. Existence of the hole. Suppose $(f, g) \in \mathcal{A}$ satisfies So. Consider the following condition (in the following, by $\operatorname{int}(X)$ we denote the set of the interior points of $X$ ):

There are closed intervals $H_{f} \subset \operatorname{int}\left(F_{1} \backslash W\right)$ and $H_{g} \subset \operatorname{int}\left(G_{1} \backslash W\right)$ with non-empty interior such that $g\left(H_{f}\right)=H_{g}$ and $f\left(H_{g}\right)=H_{f}$.

We call this property hole property and denote it by Ho (see Figure 1$)$. If $(f, g) \in \mathcal{A}$ satisfies So and Ho, then we have the following (see also Example 2 in [2]):

Proposition 2. If $(f, g) \in \mathcal{A}$ satisfies $\mathrm{So}$ and $\mathrm{Ho}$, then the (unique) minimal set $K$ of $\langle f, g\rangle_{+}$is not equal to the whole interval. More precisely, we have $K \subset I \backslash \operatorname{int}\left(H_{f} \cup H_{g}\right)$.

Proof. Suppose $K \cap \operatorname{int}\left(H_{f} \cup H_{g}\right) \neq \emptyset$. We consider the case $K \cap \operatorname{int}\left(H_{f}\right) \neq \varnothing$ (The proof of the case $K \cap \operatorname{int}\left(H_{g}\right) \neq \emptyset$ is similar so we omit it). Then we know that there exists a point $x_{0} \in \mathcal{O}_{+}(0) \cap H_{f}$. Since $x_{0} \neq 0$, we see that there exists a point $x_{1} \in \mathcal{O}_{+}(0)$ such that $x_{0}=f\left(x_{1}\right)$ or $x_{0}=g\left(x_{1}\right)$ holds. However, since $\operatorname{int}\left(H_{f}\right) \cap g(I)=\emptyset$, by Ho, the second option cannot happen. Hence we have $x_{0}=f\left(x_{1}\right) \in \operatorname{int}\left(H_{f}\right)$ and it implies $x_{1} \in$ $\operatorname{int}\left(H_{g}\right)$. By repeating this process, we continue taking the backward images of $x_{0}$ contained $\operatorname{in} \operatorname{int}\left(H_{f} \cup H_{g}\right)$. At some moment it must be equal to 0 but this is a contradiction.

2.4. Eventual expansion property. We define two maps $\mathcal{F}: F_{1} \backslash\{f(1)\} \rightarrow G_{1}$ and $\mathcal{G}: G_{1} \backslash\{g(0)\} \rightarrow F_{1}$ as follows: For $x \in F_{1} \backslash\{f(1)\}$, let $n(x)$ be the least nonnegative integer such that $g^{-n(x)}\left(f^{-1}(x)\right) \in G_{1}$ holds. Then we define a piecewise $C^{1}$ map $\mathcal{F}: F_{1} \backslash\{f(1)\} \rightarrow G_{1}$ by setting $\mathcal{F}(x):=g^{-n(x)}\left(f^{-1}(x)\right)(\mathcal{F}$ is just an "induced map" from $F_{1}$ to $G_{1}$ of the inverse system $\left(f^{-1}, g^{-1}\right)$ ). We define $\mathcal{G}$ similarly, exchanging the role of $f$ and $g$.

Then we put $R_{f}:=\mathcal{F}^{-1}\left(H_{g}\right) \subset F_{1}$ and $R_{g}:=\mathcal{G}^{-1}\left(H_{f}\right) \subset G_{1}$. We call them ruination regions. Note that by the construction $R_{f}$ is the disjoint union of infinitely many intervals 
accumulating to $f(1)$ and $R_{g}$ to $g(0)$.

Then let us consider the following property:

$\mathcal{F}$ is uniformly expanding outside $H_{f} \cup R_{f}$ and $\mathcal{G}$ is as such outside $H_{g} \cup R_{f}$.

More precisely, there exists $\mu>1$ such that at each $y \in F_{1} \backslash\left(H_{f} \cup R_{f}\right)$ (resp. $y \in$ $\left.G_{1} \backslash H_{g} \cup R_{g}\right), \mathcal{F}^{\prime}(y)>\mu$ (resp. $\mathcal{G}^{\prime}(y)>\mu$ ) where $y$ ranges over the points for which the term $\mathcal{F}^{\prime}(y)$ (resp. $\mathcal{G}^{\prime}(y)$ ) makes sense. We call this property the eventual expansion property and denote it by Ee.

In general, the property Ee may be violated by some small perturbation. However, if $f^{\prime}(0), g^{\prime}(1)<1$ then we see that Ee is a $C^{1}$-open property.

2.5. Castration on the overlap. Finally, let us consider the following property:

$$
W \subset \operatorname{int}\left(R_{f}\right) \cup \operatorname{int}\left(R_{g}\right) .
$$

We call this property the castration property and denote it by $\mathrm{Ca}$. Compared to the previous properties So, Ho and Ee, the requirement of the castration property may look artificial. Indeed, when we construct examples of IFSs, the step to obtain Ca requires involved argument compared to the other properties. See the proof of Proposition 3 in Section 4.

By the structure of $R_{f}$ and $R_{g}$, we observe the following:

REMARK 1. If Ca holds, then $g(0) \in \operatorname{int}\left(R_{f}\right)$ and $f(1) \in \operatorname{int}\left(R_{g}\right)$.

A priori, the openness of the property $\mathrm{Ca}$ is not clear, since it involves the conditions of infinitely many intervals. However, by Remark 1, together with the structure of $R_{f}$ and $R_{g}$, it can be guaranteed only by the conditions of the endpoints of finitely many intervals. As a consequence, we see that $\mathrm{Ca}$ is a $C^{0}$-open property in $\mathcal{A}$.

2.6. Main statement. We denote the set of pairs of diffeomorphisms in $\mathcal{A}$ satisfying the conditions So, $\mathrm{Ho}$, Ee and $\mathrm{Ca}$ by $\mathcal{C}$. Now, we state our main result.

THEOREM. If $(f, g)$ is in $\mathcal{C}$ then the (unique) minimal set of $\langle f, g\rangle_{+}$is homeomorphic to the Cantor set.

Later we also prove the following:

PRoposition 3. In $\mathcal{A} \subset\left(\operatorname{Diff}_{\mathrm{im}}^{1}(I)\right)^{2}$, the set $\mathcal{C}$ has non-empty interior (with respect to the relative topology on $\mathcal{A}$ induced by the $C^{1}$-topology on $\left.\left(\operatorname{Diff}_{\mathrm{im}}^{1}(I)\right)^{2}\right)$.

As a corollary, we see that our example can be taken $C^{\infty}$ or $C^{\omega}$.

Remark 2. By Proposition 2, for $(f, g) \in \mathcal{A}$ satisfying the condition So and Ho, we know that the action of $\langle f, g\rangle_{+}$is not minimal. However, in general the author does not know if the minimal set is homeomorphic to the Cantor set or not. 


\section{Proof of Theorem}

In this section, we give the proof of Theorem. We fix $(f, g) \in \mathcal{C}$ and consider its unique minimal set $K$.

3.1. Characterization of the Cantor set. We first remember the famous characterization of the Cantor set: If a topological space is compact, metrizable, perfect and totally disconnected, then it is homeomorphic to the Cantor set (see for example [5]). In our setting, it is clear that $K$ satisfies the first and the second properties. The perfectness of the minimal set can be seen easily by the definition of the minimal set. Thus we only need to prove the totally disconnectedness of $K$. For that, since we work on one dimensional setting, we only need to prove the emptiness of the interior. We state it in a more precise way.

REMARK 3. For the proof of Theorem, we only need to prove the following: For every $x \in K$ and every non-empty open interval $J \subset I$ containing $x$, there exists a non-empty interval $L \subset J$ such that $L \cap K=\emptyset$.

3.2. Taking the backward image of the orbit. First, we start from some simple observations.

LEMMA 1. Let $J \subset I$ be a non-empty open interval.

- If $J \subset \operatorname{int}\left(F_{1} \backslash W\right)\left(\right.$ resp. $\left.J \subset \operatorname{int}\left(G_{1} \backslash W\right)\right)$ and $J \cap K \neq \emptyset$, then $\mathcal{F}(J) \cap K \neq \emptyset$ (resp. $\mathcal{G}(J) \cap K \neq \emptyset$ ).

- Suppose $J \subset \operatorname{int}(W)$ and $J \cap K \neq \emptyset$. Then, if $J \subset \operatorname{int}\left(R_{f}\right)\left(\right.$ resp. $J \subset \operatorname{int}\left(R_{g}\right)$ ), we have $\mathcal{G}(J) \cap K \neq \emptyset$ (resp. $\mathcal{F}(J) \cap K \neq \emptyset)$.

PROOF. For the proof, one important observation is the following: If $p \in F_{1} \backslash W$ belongs to $\mathcal{O}_{+}(0)$, then $\mathcal{F}(p) \in \mathcal{O}_{+}(0)$. Indeed, in general, for given $p \in \mathcal{O}_{+}(0)$ we have either $f^{-1}(p) \in \mathcal{O}_{+}(0)$ or $g^{-1}(p) \in \mathcal{O}_{+}(0)$ (both condition may hold simultaneously). If $p \in F_{1} \backslash W$, the second option cannot hold. Thus we see that $f^{-1}(p) \in \mathcal{O}_{+}(0)$. Then, fix $k \geq 1$ so that $f^{-1}(p) \in G_{k}$ holds. By repeating the similar reasoning, we can prove that $\left(g^{-j+1} \circ f^{-1}\right)(p) \in \mathcal{O}_{+}(0)$ for every $j$ with $1 \leq j \leq k$ (by the induction of $j$ ). In particular, we have $\left(g^{-k+1} \circ f^{-1}\right)(p) \in \mathcal{O}_{+}(0)$, which is equal to $\mathcal{F}(p)$ by definition.

Let us start the proof of the first item. By the symmetry, we only consider the case $J \subset \operatorname{int}\left(F_{1} \backslash W\right)$ (and omit the case $J \subset \operatorname{int}\left(G_{1} \backslash W\right)$ ). Suppose $J \subset \operatorname{int}\left(F_{1} \backslash W\right)$ satisfies $J \cap K \neq \emptyset$. By the assumption and the fact $K=\overline{\mathcal{O}_{+}(0)}$, there exists a point $p \in \mathcal{O}_{+}(0) \cap J$. By above observation, we see $\mathcal{F}(p) \in K$. It implies $\mathcal{F}(J) \cap K \neq \emptyset$.

Let us consider the proof of the second item. Again by the symmetry between $f$ and $g$, we only treat the case where $J \subset \operatorname{int}\left(R_{f}\right)$ and omit the case $J \subset \operatorname{int}\left(R_{g}\right)$. Suppose $J \subset \operatorname{int}\left(R_{f}\right)$ and there exists a point $p \in J \cap \mathcal{O}_{+}(0)$. Then, as is the previous discussion, one of the following options holds: $\mathcal{F}(p) \in \mathcal{O}_{+}(0)$ or $\mathcal{G}(p) \in \mathcal{O}_{+}(0)$. However, the condition $p \in R_{f}$ implies $\mathcal{F}(p) \in \operatorname{int}\left(H_{f}\right)$ and this, together with Proposition 2, prohibits the first option. Thus we have $\mathcal{G}(p) \in \mathcal{O}_{+}(0)$, which implies what we claimed. 
In the similar way, we have the following.

Lemma 2. If $J \subset \operatorname{int}\left(R_{f} \cap R_{g}\right)$, then $J \cap K=\emptyset$.

Proof. Suppose $J \subset \operatorname{int}\left(R_{f} \cap R_{g}\right)$ and $J \cap K \neq \emptyset$. By assumption, we have $J \subset$ $\operatorname{int}\left(R_{f}\right)$. By the second item of Lemma 1, we see $\mathcal{G}(J) \cap K \neq \emptyset$. However, $J \subset \operatorname{int}\left(R_{g}\right)$ implies $\mathcal{G}(J) \subset \operatorname{int}\left(H_{g}\right)$, which contradicts to the fact that $H_{g} \cap K=\emptyset$ (see Proposition 2).

We prepare one definition. For $(f, g) \in \mathcal{C}$, we define

$$
B_{f}:=\partial\left(H_{f} \cup\left(R_{f} \cap R_{g}\right)\right) \cup \partial F_{1}, \quad B_{g}:=\partial\left(H_{g} \cup\left(R_{f} \cap R_{g}\right)\right) \cup \partial G_{1},
$$

where $\partial X$ denotes the boundary of $X$. Then we have the following.

LEMMA 3. If $J \subset I$ is an open interval with $J \cap B_{f} \neq \emptyset$ (resp. $\left.J \cap B_{g} \neq \emptyset\right)$, then there exists a non-empty open interval $U \subset J$ with $U \cap K=\emptyset$.

Proof. Take $J \subset I$ with $J \cap B_{f} \neq \emptyset$. We divide our situation into four cases: $J \cap H_{f} \neq \emptyset, J \cap \partial\left(R_{f} \cap R_{g}\right) \neq \emptyset, f(1) \in J$ or $f^{2}(1) \in J$.

For the first case, remember Proposition 2: It implies that every non-empty open set $U \subset J \cap \operatorname{int}\left(H_{f}\right)$ is disjoint from $K$. Thus one of such $U$ gives us the desired set.

In the second case, using the castration property (that is, $W \subset \operatorname{int}\left(R_{f}\right) \cup \operatorname{int}\left(R_{g}\right)$ ), we see that there exists a non-empty open set $U \subset J \cap\left(R_{f} \cap R_{g}\right)$. By Lemma 2, this $U$ gives the desired set. The third case is reduced to the second case by Remark 1 and the fact that $R_{f}$ accumulates to $f(1)$.

In the last case, consider $f^{-1}(J)$. Then it contains $f(1)$. Thus by the previous argument, we can take $U \subset f^{-1}(J)$ with $U \cap K=\emptyset$. Then, by repeating the similar argument as Lemma 1, we see that $f(U) \subset J$ is the desired interval.

3.3. Induction: The proof of Theorem. Now we start the proof of Theorem. Remember that to prove the Theorem we only need to prove the statement in Remark 3 . The idea of the proof is using Lemma 1,2 and 3 inductively to examine the possible past behavior of $J$.

We start the proof for $J$ satisfying $J \cap\left(F_{1} \cup G_{1}\right) \neq \emptyset$. The other case can be reduced to this specific case easily. We will discuss it later.

Proof of Theorem For $J$ WITH $J \cap\left(F_{1} \cup G_{1}\right) \neq \emptyset$. Given $J \subset I$ with $J \cap\left(F_{1} \cup\right.$ $\left.G_{1}\right) \neq \emptyset$, we define a $C^{1}$-map $\tau: J \rightarrow I$ which is a diffeomorphism on its image and a non-empty open interval $U \subset \tau(J)$. After that we will see that $L:=\tau^{-1}(U)$ is the claimed interval in Remark 3. For that, we define a finite sequence of maps $\left(\tau_{n}\right)$ and intervals $\left(J_{n}\right)$ inductively as follows.

We put $\tau_{0}:=\mathrm{id}$ and $J_{0}:=J$. Suppose that we have defined $\tau_{k}$ and $J_{k}$ ( $k$ is some non-negative integer). Then we proceed as follows: First, if $J_{k} \cap\left(B_{f} \cup B_{g}\right) \neq \emptyset$, then take the interval $U \subset J_{k}$ by applying Lemma 3 letting $J=J_{k}$, put $\tau=\tau_{k}$ and finish the 
construction. Suppose not. Then we have five possibilities: $J_{k} \subset H_{f}, J_{k} \subset H_{g}, J_{k} \subset W$, $J_{k} \subset F_{1} \backslash\left(H_{f} \cup W\right)$ or $J_{k} \subset G_{1} \backslash\left(H_{g} \cup W\right)$. We proceed as follows.

- In the first (resp. second) case, set $\tau=\tau_{k}, U=J_{k}$ and finish the construction. Note that by Proposition 2, we see that $U \cap K=\emptyset$.

- Suppose $J_{k} \subset W$. In this case, we know that $J$ is contained in either $\operatorname{int}\left(R_{f} \backslash R_{g}\right)$ or $\operatorname{int}\left(R_{g} \backslash R_{f}\right)$ (remember that we are under the assumption $J \cap\left(B_{f} \cup B_{g}\right)=\emptyset$ ).

- If $J \subset \operatorname{int}\left(R_{f} \backslash R_{g}\right)$, then put $\tau_{k+1}:=\mathcal{G} \circ \tau_{k}, J_{k+1}:=\mathcal{G}\left(J_{k}\right)$ and continue the construction.

- Otherwise (that is, if $J \subset \operatorname{int}\left(R_{g} \backslash R_{f}\right)$ ), then put $\tau_{k+1}:=\mathcal{F} \circ \tau_{k}, J_{k+1}:=\mathcal{F}\left(J_{k}\right)$ and continue the construction.

- In the fourth (resp. fifth) case, set $\tau_{k+1}:=\mathcal{F} \circ \tau_{k}$ (resp. $\left.\tau_{k+1}:=\mathcal{G} \circ \tau_{k}\right), J_{k+1}:=\mathcal{F}\left(J_{k}\right)$, (resp. $J_{k+1}:=\mathcal{G}\left(J_{k}\right)$ ) and continue the construction.

Note that, by eventual expansion property, this process finishes in finite steps: We can check that every time we apply $\mathcal{F}$ (resp. $\mathcal{G}$ ) to $J_{k}$ in the above construction, $J_{k}$ is outside $H_{f} \cap R_{f}$ (resp. $H_{g} \cap R_{g}$ ). Thus for given $J$, we can take $\tau$ and $U \subset \tau(J)$. Note that, by construction, $\left.\tau\right|_{J}: J \rightarrow I$ is a diffeomorphism on its image and $U \subset I$ satisfies $U \cap K=\emptyset$.

Then consider the non-empty open interval $\tau^{-1}(U) \subset J$. We claim that $\tau^{-1}(U) \cap K=$ $\emptyset$. Indeed, suppose $\tau^{-1}(U) \cap K \neq \emptyset$. This implies $\tau^{-1}(U) \cap \mathcal{O}_{+}(0) \neq \emptyset$. However, applying Lemma 1 or 2 to each option of the construction of $\tau_{1}$, we see that $\tau_{1}\left(\tau^{-1}(U)\right)$ contains a point of $\mathcal{O}_{+}(0)$. Then by induction we conclude that $\tau_{j}\left(\tau^{-1}(U)\right) \cap \mathcal{O}_{+}(0) \neq \varnothing$ for every $j$. In particular, we see that $\tau\left(\tau^{-1}(U)\right)=U$ contains some point of $K$, which contradicts to the choice of $U$.

We give the proof for the case where $J$ is outside $F_{1} \cup G_{1}$, which completes the proof of Theorem.

Proof of TheOREM FOR $J$ NOT CONTAINEd In $F_{1} \cup G_{1}$. In this case, we know that $J$ is contained in $f(I) \backslash F_{1}$ or $g(I) \backslash G_{1}$. By the symmetry, we only need to consider the first case.

By shrinking $J$ if necessary, we can assume that there exists $N \geq 2$ such that $J \subset F_{N}$. Thus we have $f^{-N+1}(J) \subset F_{1}$. Applying the conclusion of the previous case, we take a non-empty open set $U \subset f^{-N+1}(J)$ such that $U \cap K=\emptyset$. Then, consider $f^{N-1}(U) \subset J$. We claim that $f^{N-1}(U) \cap K=\emptyset$ (which implies what we want to prove).

Indeed, if not, there exists a point $x \in f^{N-1}(U) \cap \mathcal{O}_{+}(0)$. Then, repeating the argument used in the proof of Lemma 1, together with the single overlapping property So, we see that $f^{N-1-j}(U) \cap K \neq \emptyset$ for every $0 \leq j \leq N-1$ (by the induction of $j$ ). In particular it implies that $U \cap K \neq \emptyset$, but this is a contradiction. 


\section{Construction of the example}

In this section, we prove Proposition 3, that is, we describe how we construct the example.

4.1. Start of the construction and first modification. We start from the system

$$
f_{*}(x)=(1 / 2) x, \quad g_{*}(x)=(1 / 2) x+1 / 2 .
$$

Then, put $p:=1 / 3, q:=2 / 3$ and take intervals $J_{p}, J_{q}$ centered at $p, q$ respectively, with sufficiently small diameter (for example, $\left|J_{p}\right|=\left|J_{q}\right|=1 /(100)$ is enough).

We modify $f_{*}$ and $g_{*}$ to get the example. Every modification except the last one is performed keeping the symmetry of the graph with respect to the diagonal: $1-f(1-x)=$ $g(x)$ holds for every $x \in[0,1]$. First we modify $f_{*}$ and $g_{*}$ to $C^{1}$-maps $f_{0}$ and $g_{0}$ respectively, keeping them in $\operatorname{Diff}_{\text {im }}^{1}$ so that the following holds:

- $f_{0}=f_{*}$ outside $J_{p}$ and $g_{0}=g_{*}$ outside $J_{q}$.

- There are intervals $J_{p}^{\prime} \subset \operatorname{int}\left(J_{p}\right), J_{q}^{\prime} \subset \operatorname{int}\left(J_{q}\right)$ such that $J_{q}^{\prime} \subset \operatorname{int}\left(g_{0}\left(J_{p}^{\prime}\right)\right)$ and $J_{p}^{\prime} \subset$ $\operatorname{int}\left(f_{0}\left(J_{q}^{\prime}\right)\right)$.

Such a modification can be done just by modifying local behavior around of $f_{0}$ (resp. $g_{0}$ ) around $q$ (resp. $p$ ) expanding.

Then we have the following:

Proposition 4. There exist intervals $H_{p} \subset \operatorname{int}\left(J_{p}\right)$ and $H_{q} \subset \operatorname{int}\left(J_{q}\right)$ such that $J_{p}^{\prime} \subset \operatorname{int}\left(H_{p}\right), J_{q}^{\prime} \subset \operatorname{int}\left(H_{q}\right)$ and $f_{0}\left(H_{q}\right)=H_{p}, g_{0}\left(H_{p}\right)=H_{q}$.

Proof. By the definition, we have $f_{0} \circ g_{0}\left(J_{p}\right) \subset \operatorname{int}\left(J_{p}\right)$. So, $H_{p}:=\cap_{n \geq 0}\left(f_{0} \circ\right.$ $\left.g_{0}\right)^{n}\left(J_{p}\right)$ is a closed interval contained in $\operatorname{int}\left(J_{p}\right)$. We also know that it cannot be a point, since it contains $J_{p}^{\prime}$. Furthermore, by the definition we can see that $\left(f_{0} \circ g_{0}\right)\left(H_{p}\right)=H_{p}$. Then $H_{q}:=g_{0}\left(H_{p}\right)$ and $H_{p}$ are the desired intervals.

4.2. Second modification. From $\left(f_{0}, g_{0}\right)$, we construct a one-parameter family of IFSs $\left(f_{\varepsilon}, g_{\varepsilon}\right)$ as follows.

We fix a small positive number $k\left(k<1 /(100)\right.$ is enough) and modify $f_{0}, g_{0}$ to $\left(f_{\varepsilon}, g_{\varepsilon}\right)$, where $\varepsilon$ is some non-negative small real number, keeping being continuous and such that the following holds:

- On $(1-k, 1), f_{\varepsilon}$ is an affine map with slope $(1 / 2)+\varepsilon$,

- on $(0, k), g_{\varepsilon}$ is an affine map with slope $(1 / 2)+\varepsilon$,

- and keep the other dynamics intact.

Note that there exists a small real number $\delta>0$ so that for every $\varepsilon \in(0, \delta)$ the pair $\left(f_{\varepsilon}, g_{\varepsilon}\right)$ satisfies the condition $\mathcal{A}$, the single overlapping property So and the hole property Ho. Hence the notions such as $F_{i}, G_{i}, W, R_{f_{\varepsilon}}$ and $R_{g_{\varepsilon}}$ make sense for $\left(f_{\varepsilon}, g_{\varepsilon}\right)$. These objects vary as $\varepsilon$ varies. To clarify the dependence, we put $\varepsilon$ to them. For example, $F_{2}^{\varepsilon}$ denotes the 
interval $F_{2}$ for $\left(f_{\varepsilon}, g_{\varepsilon}\right)$. Note that if $k$ is chosen sufficiently small then $H_{p}$ and $H_{q}$ do not depend on $\varepsilon$. Thus we do not put $\varepsilon$ to them.

In $W^{\varepsilon}$, we define two sequences of intervals. For $n \geq 0$, we define $P_{n}^{\varepsilon}$ as follows:

$$
P_{n}^{\varepsilon}:=\left\{x \in W^{\varepsilon} \mid f_{\varepsilon}^{-n} \circ g_{\varepsilon}^{-1}(x) \in H_{p}\right\} .
$$

Similarly, we define $Q_{n}^{\varepsilon}$ as follows:

$$
Q_{n}^{\varepsilon}:=\left\{x \in W^{\varepsilon} \mid g_{\varepsilon}^{-n} \circ f_{\varepsilon}^{-1}(x) \in H_{q}\right\} .
$$

For fixed $\varepsilon$, the interval $P_{n}^{\varepsilon}, Q_{n}^{\varepsilon}$ may be empty, but for large $n$ it is not empty. Note that $\left\{Q_{n}^{\varepsilon}\right\}$ accumulates to $f_{\varepsilon}(1),\left\{P_{n}^{\varepsilon}\right\}$ to $g_{\varepsilon}(0)$. We also have $R_{g_{\varepsilon}}^{\varepsilon}=\amalg P_{n}^{\varepsilon}$ and $R_{f_{\varepsilon}}^{\varepsilon}=\amalg Q_{n}^{\varepsilon}$.

Then we define $C \subset(0, \delta)$ as follows:

$$
C:=\left\{\varepsilon \in(0, \delta) \mid g_{\varepsilon}(0) \in R_{f_{\varepsilon}}^{\varepsilon}, f_{\varepsilon}(1) \in R_{g_{\varepsilon}}^{\varepsilon}\right\} .
$$

Now we prove the following:

PROPOSITION 5. C is not empty.

PROOF. Consider the set $H_{q}^{\prime}:=\bigcup_{n \geq 0} g_{\varepsilon}^{n}\left(H_{q}\right)$. This is a disjoint union of intervals converging to 1 , and we can see that $H_{q}^{\prime}$ does not depend on $\varepsilon$.

Because of the symmetry, the condition $\varepsilon \in C$ is equivalent to $f_{\varepsilon}^{-1}\left(g_{\varepsilon}(0)\right) \in H_{q}^{\prime}$. So let us investigate how $f_{\varepsilon}^{-1}\left(g_{\varepsilon}(0)\right)$ varies when $\varepsilon$ varies. By the definition, we can see that for $\varepsilon$ near $0, f_{\varepsilon}^{-1}\left(g_{\varepsilon}(0)\right)$ is monotone increasing as $\varepsilon$ decreases and it converges to 1 when $\varepsilon \searrow 0$. Thus, for sufficiently large $n$, there is an interval $C_{n} \subset C$ such that for $\varepsilon \in C_{n}$ we have $f_{\varepsilon}^{-1}\left(g_{\varepsilon}(0)\right) \in g_{\varepsilon}^{n}\left(H_{q}\right)$. In particular, $C$ is not empty.

We fix a parameter $\alpha \in C$ and perform the last modification to $\left(f_{\alpha}, g_{\alpha}\right)$ as follows. For this IFS, there exists $n_{0}$ such that $g_{\alpha}(0) \in P_{n_{0}}^{\alpha}$ holds by definition. By the symmetry, note that we also have $f_{\alpha}(1) \in Q_{n_{0}}^{\alpha}$. We take an interval $\tilde{P} \subset W^{\alpha}$ such that $W^{\alpha} \subset \tilde{P} \cup Q_{n_{0}}^{\alpha}$ holds. Then we pick up a $C^{1}$-diffeomorphism $\gamma: W^{\alpha} \rightarrow W^{\alpha}$ satisfying the following:

- There exists an interval $\tilde{P}^{\prime} \subset W^{\alpha}$ which contains $\tilde{P}$ such that $\gamma\left(\tilde{P}^{\prime}\right) \subset P_{n_{0}}^{\alpha}$.

- There exists a constant $\mu^{\prime}>1$ such that $\left.\gamma^{\prime}\right|_{W \backslash \tilde{P}^{\prime}}>\mu^{\prime}$.

- $\gamma^{\prime}\left(g_{\alpha}(0)\right)=1$.

The construction of $\gamma$ is not difficult, so we omit the detail (see Figure 2).

Then we modify $f_{\alpha}$ to $\tilde{f}_{\alpha}$ so that the following holds:

- $\left.\left(\tilde{f}_{\alpha}\right)^{-1}\right|_{W}=\left(f_{\alpha}\right)^{-1} \circ \gamma$.

- $\left.\left(\tilde{f}_{\alpha}\right)^{-1}\right|_{f_{\alpha}(I) \backslash W}=\left(f_{\alpha}\right)^{-1}$.

Note that the last condition of $\gamma$ guarantees that the resulted map is $C^{1}$.

Then we claim the following: 


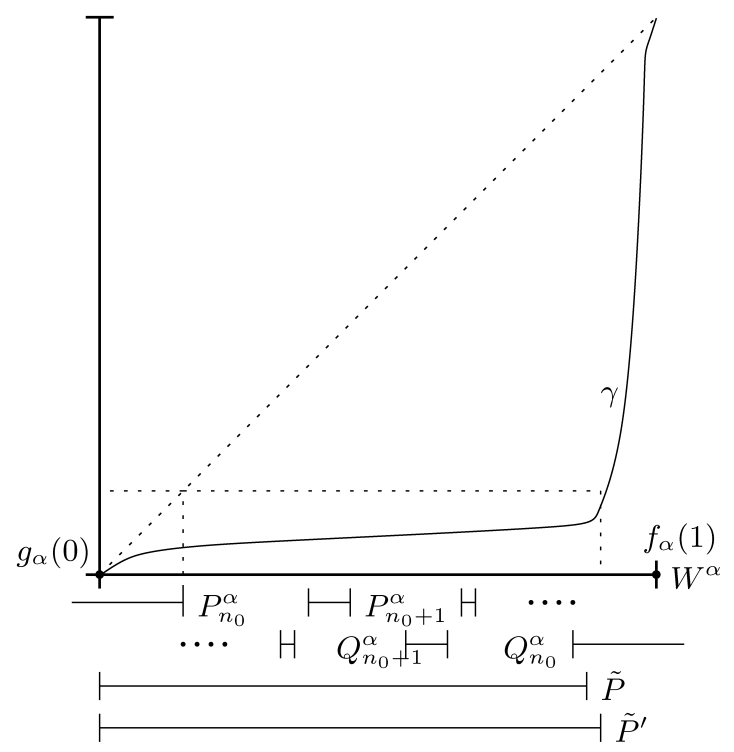

FIGURE 2. For the construction of $\gamma$

Proposition 6. This $\left(\tilde{f}_{\alpha}, \tilde{g}_{\alpha}\right)$ satisfies properties So, Ho, Ee and Ca.

Proof. It is easy to check the properties So, Ho hold for $\left(\tilde{f}_{\alpha}, \tilde{g}_{\alpha}\right)$. To check the castration property, let us check that we have $W^{\alpha} \subset R_{\tilde{f}_{\alpha}} \cup R_{\tilde{g}_{\alpha}}$ for $\left(\tilde{f}_{\alpha}, \tilde{g}_{\alpha}\right)$. Since the only difference between $\left(\tilde{f}_{\alpha}, \tilde{g}_{\alpha}\right)$ and $\left(f_{\alpha}, g_{\alpha}\right)$ is the behavior of $\left(\tilde{f}_{\alpha}\right)^{-1}$ on $W^{\alpha}$, we see that $R_{\tilde{g}_{\alpha}}=R_{g_{\alpha}}$. Furthermore, by the modification from $f_{\alpha}$ to $\tilde{f}_{\alpha}$, we have $\tilde{P}^{\prime} \subset R_{\tilde{f}_{\alpha}}$. Thus, together with the fact $W^{\alpha} \subset \tilde{P} \cup Q_{n_{0}}^{\alpha}$, we see the castration property for $\left(\tilde{f}_{\alpha}, \tilde{g}_{\alpha}\right)$.

Finally we check the eventual expansion property Ee of $\left(\tilde{f}_{\alpha}, \tilde{g}_{\alpha}\right)$. The uniform expansion of $\mathcal{G}$ is clear from the construction. To check the uniform expansion of $\mathcal{F}$, the problem is that we composed $\gamma$ with $f_{\alpha}^{-1}$ and it may (and does) have a contracting behaviors on some domain of definition of $\mathcal{F}$. However, by construction such regions are contained in $\tilde{P}^{\prime}$ and hence in $R_{\tilde{f}_{\alpha}}$. Thus we also have the expansion property of $\mathcal{F}$.

\section{A. Another example}

After finding the previous example, Masayuki Asaoka showed me another type of example. In this appendix we present it.

We fix a small number $\varepsilon>0$ (in practice, $\varepsilon=1 /(100)$ is enough) and another number $\lambda>0$ smaller than $1 / 2$. Then we define as follows: 


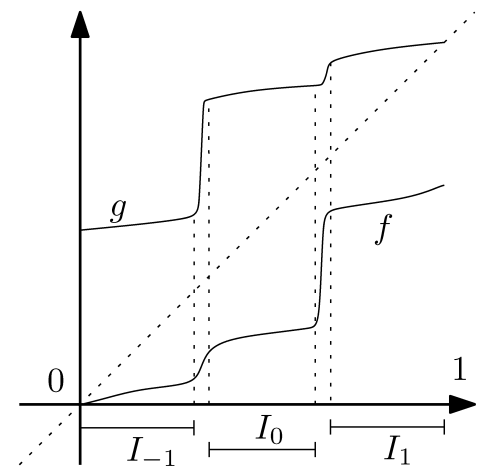

FIGURE 3. An example of IFS in the Appendix

$$
I_{-1}:=[0,1 / 3-\varepsilon], \quad I_{0}:=[1 / 3+\varepsilon, 2 / 3-\varepsilon], \quad I_{1}:=[2 / 3+\varepsilon, 1] .
$$

Then take $(f, g) \in \mathcal{A}$ such that the following holds:

- $f\left(I_{-1}\right) \subset I_{-1}, \quad f\left(I_{0}\right) \subset \operatorname{int}\left(I_{-1}\right), \quad f\left(I_{1}\right) \subset \operatorname{int}\left(I_{0}\right)$.

- $g\left(I_{1}\right) \subset I_{1}, \quad g\left(I_{0}\right) \subset \operatorname{int}\left(I_{1}\right), \quad g\left(I_{-1}\right) \subset \operatorname{int}\left(I_{0}\right)$.

- $\left.f^{\prime}\right|_{I_{-1} \cup I_{0} \cup I_{1}},\left.g^{\prime}\right|_{I_{-1} \cup I_{0} \cup I_{1}}<\lambda$.

We call the first and the second conditions inclusion property, and the last condition strong uniform contraction property.

The construction of such pair of maps in $\mathcal{A}$ is not difficult (see the graph in Figure 3), so we skip the detail. Note that these properties are open in $\mathcal{A}$ (the conditions $f\left(I_{-1}\right) \subset$ $I_{-1}, g\left(I_{1}\right) \subset I_{1}$ are not open in $\left(\operatorname{Diff}_{\mathrm{im}}^{1}\right)^{2}$, but in $\mathcal{A}$ they are).

We claim the following:

Proposition 7. The (unique) minimal set $K$ for above $(f, g)$ is homeomorphic to the Cantor set.

For the proof, remember that we only need to prove the emptiness of the interior of the minimal set (see Section 3.1). For that we prove that the Lebesgue measure of $K$ is zero. We denote the Lebesgue measure on $I$ by $\mu$.

First, we define the sequence of compact sets $\left(\Lambda_{n}\right)$ as follows:

- $\Lambda_{0}:=I_{-1} \cup I_{0} \cup I_{-1}$.

- $\Lambda_{k+1}:=f\left(\Lambda_{k}\right) \cup g\left(\Lambda_{k}\right)$.

By the inclusion property of $f$ and $g$, we know that $\left(\Lambda_{n}\right)$ is a nested sequence of compact sets. Thus $\Lambda:=\cap \Lambda_{n}$ is a non-empty compact set. Furthermore, by definition we see that $\mathcal{O}_{+}(0) \subset \Lambda$. Thus we have $K \subset \Lambda$ (indeed, we can prove the equality between $K$ and $\Lambda$ but since we do not need it we omit it).

Then we claim the following: 
Claim 1. $\mu(\Lambda)=0$, in particular $\mu(K)=0$.

PROOF. We prove that for every $n \geq 0$ we have $\mu\left(\Lambda_{n}\right) \leq(2 \lambda)^{n}$, which concludes $\mu(\Lambda)=0$ for $\lambda<1 / 2$.

The case $n=0$ is clear. Suppose the inequality is true for $n=k$ (where $k$ is some non-negative integer). First, by definition we have

$$
\mu\left(\Lambda_{k+1}\right) \leq \mu\left(f\left(\Lambda_{k}\right)\right)+\mu\left(g\left(\Lambda_{k}\right)\right) .
$$

Then, by uniform contracting property we have

$$
\mu\left(f\left(\Lambda_{k}\right)\right)<\lambda \mu\left(\Lambda_{k}\right), \quad \mu\left(g\left(\Lambda_{k}\right)\right)<\lambda \mu\left(\Lambda_{k}\right) .
$$

Thus we have

$$
\mu\left(\Lambda_{k+1}\right) \leq(2 \lambda) \mu\left(\Lambda_{k}\right)<(2 \lambda)^{k+1},
$$

which completes the proof.

REMARK 4. The first example and the second one are conceptually different. The first example is constructed by investigating the past behavior of the dynamics, while the second one is constructed by investigating the future behavior.

Acknowledgment. This research is supported by the Aihara Project, the FIRST program from JSPS, initiated by CSTP. The author is thankful to Masayuki Asaoka, Kengo Shimomura, Hiroki Sumi and Dmitry Turaev for helpful conversations.

\title{
References
}

[ 1] P. BARRientos and A. RaibeKas, Dynamics of iterated function systems on the circle close to rotations, preprint, accepted, to appear in Ergod. Th. \& Dynam. Sys.

[2] K. Shinohara, On the minimality of semigroup actions on the interval which are $C^{1}$-close to the identity, preprint, accepted, to appear Proc. Lond. Math. Soc.

[ 3 ] A. NAVAs, Groups of circle diffeomorphisms, Translation of the 2007 Spanish edition. Chicago Lectures in Mathematics. University of Chicago Press, Chicago, IL, 2011. xviii+290 pp.

[ 4 ] H. SUMI, Interaction cohomology of forward or backward self-similar systems, Adv. Math. 222 (2009), no. 3, 729-781.

[ 5 ] S. Willard, General topology, Dover Publications, Inc., Mineola, NY, 2004. xii+369 pp.

\author{
Present Address: \\ DePARTMENT OF MATHEMATICS AND INFORMATION SCIENCES, \\ TOKYO METROPOLITAN UNIVERSITY, \\ 1-1, Minami-OS AWA HACHIOJi, TOKYO, 192-0397 JAPAN. \\ e-mail: herrsinon@07.alumni.u-tokyo.ac.jp
}

